\title{
Diffraction Regimes of Single Holes
}

\author{
J.-M. Yi ${ }^{1}{ }^{\text {A. }}$ Cuche, ${ }^{1}$ F. de León-Pérez,${ }^{2,3}$ A. Degiron, ${ }^{1}$ E. Laux, ${ }^{1}$ E. Devaux,${ }^{1}$ C. Genet,,${ }^{1}$ J. Alegret, ${ }^{3}$ \\ L. Martín-Moreno, ${ }^{3}$ and T. W. Ebbesen ${ }^{1}$ \\ ${ }^{1}$ ISIS \& icFRC, University of Strasbourg and CNRS, 8, allée Gaspard Monge, 67000 Strasbourg, France \\ ${ }^{2}$ Centro Universitario de la Defensa de Zaragoza, Carretera de Huesca, E-50090 Zaragoza, Spain \\ ${ }^{3}$ Instituto de Ciencia de Materiales de Aragón and Departamento de Física de la Materia Condensada, \\ CSIC-Universidad de Zaragoza, E-50009 Zaragoza, Spain
}

(Received 2 February 2012; published 12 July 2012)

\begin{abstract}
We investigate both experimentally and theoretically the far-field diffraction patterns of single circular apertures as a function of their diameters $d$ and at a given illumination wavelength $\lambda$. We observe the transition between the well-known pseudoscalar regime of large holes $(d \gg \lambda)$ and the less-known vectorial regime of subwavelength ones $(d \ll \lambda)$. Four different diffraction regimes are identified for different $d / \lambda$ regions, each one with its polarization dependence. A thorough comparison with a theoretical model, which takes into account both finite hole size and the dielectric properties of the metal, allows us to explain and understand the physical processes leading to this behavior. Our results reveal the subtle interplay between two competing factors, one related to polarization symmetries associated with surface-plasmon excitations and the other originating in the coupling of the field to the waveguide mode of the aperture.
\end{abstract}

DOI: 10.1103/PhysRevLett.109.023901

PACS numbers: 42.79.Ag, 42.25.Fx, 42.25.Ja, 73.20.Mf

Single subwavelength apertures play an important part in the most advanced techniques in nano-optics. Their optical behavior is central to high resolution near-field scanning microscopies [1], extraordinary optical transmission phenomena [2,3], surface-plasmon assisted light beaming [4], fluorescence correlation spectroscopy [5,6], optical trapping [7], and recent magnetometry strategies [8]. Still, the diffractive properties of subwavelength holes in real metallic films, including the plasmonic contribution, have continually raised fundamental questions since their first scientific documentation by Grimaldi in the 17th century and many more recent experimental and theoretical studies [9-15]. The observed diffraction patterns of circular apertures large compared to the wavelength ( $k r \gg 1$, where $k$ is the wave number of the incident wave and $r$ the radius of the hole) can be explained by Kirchhoff's scalar theory. Accordingly, the normalized angular intensity in the far field (Fraunhofer regime) is given by $I_{\mathrm{Kir}}(\theta)=\left(2 J_{1}(\Phi) / \Phi\right)^{2}$, where $J_{1}(\Phi)$ is the Bessel function of the first order and $\Phi=k r \sin (\theta)$, with $\theta$ the observation angle. This model can be extended up to $k r \sim 1$ by taking into account the polarization dependence of the angular emission [16]. In the opposite subwavelength case, $k r \ll 1$, the basic assumptions of Kirchhoff's approach no longer hold, as pointed out by Bethe and Bouwkamp [17,18]. In this regime, they were able to provide a rigorous analysis of Maxwell's equations in an infinitesimally thin perfect electric conductor (PEC) [17]. Bethe showed that the diffraction pattern of the hole can ultimately be calculated from the field radiated by two crossed dipoles associated with the aperture, one electric (P) normal to and one magnetic $(\mathbf{M})$ in the aperture plane.
In the ideal case where a plane wave impinges a circular aperture at normal incidence, this leads to a simple description of the normalized angular distributions in the far field with $I_{\|}(\theta)=1$ and $I_{\perp}(\theta)=\cos ^{2}(\theta)$ when scanning, respectively, in a plane parallel and perpendicular to the incident polarization. Since the work of Bethe, the transmission through finite-sized holes in PEC films has been extensively studied theoretically [19,20]. Interestingly, experimental observation of subwavelength holes in the optical regime using real metal films, with finite dielectric constants and finite thicknesses (and thus plasmon resonance), shows that their diffraction cannot be accounted for by Bethe's model [10-15]. In addition some of these studies are further complicated by the geometry of the experiment with the aperture at the apex of a metal-covered optical fiber in optically thin conditions [9,21,22].

This led us to design a set of experiments where the diffraction of a circular aperture in a planar opaque metal film could be monitored continuously from $k r \gg 1$ to $k r \ll 1$ in order to clarify the optical properties of subwavelength holes and, in particular, observe the transition between these two regimes. In parallel, simple analytical expressions have been developed using the theoretical framework of the modal expansion formalism, which are in excellent agreement with the experimental results. As we report in this Letter, four diffraction regimes are identified for different values of $k r$, each with its polarization dependence. By comparing these results with the model, we can explain the existence of these regimes by the subtle interplay of two competing factors, one related to polarization symmetries associated with surface-plasmon (SP) excitations, which is independent of the hole size, and the 


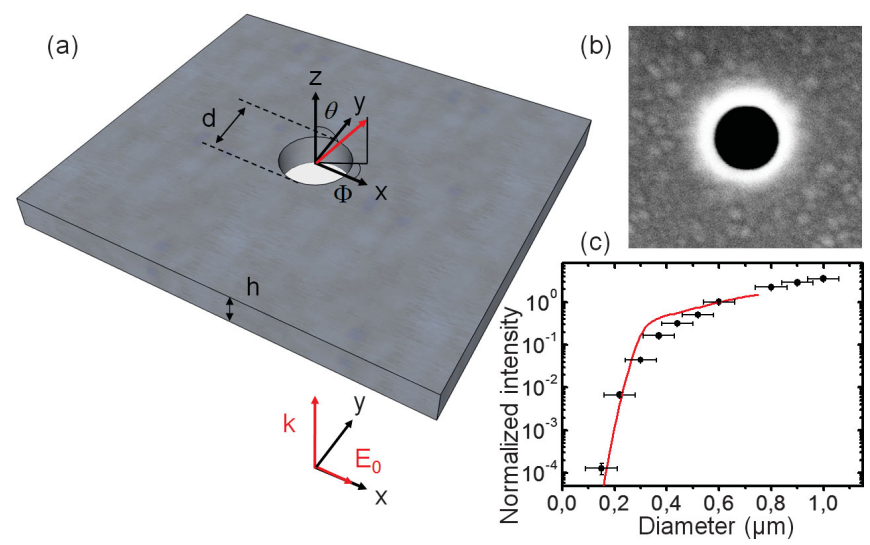

FIG. 1 (color). (a) Scheme of the single hole geometry. (b) SEM image of a single hole with a diameter $d=220 \mathrm{~nm}$. (c) Transmitted intensity at $\theta=0^{\circ}$ for a single hole in Ag illuminated at normal incidence, as a function of hole diameter (film thickness $h=300 \mathrm{~nm}$ ). Experimental data are shown as black circles (including error bars on the measured angular distributions and hole diameters) while calculations performed using the coupled mode method are rendered by the solid red line.

other originating in the coupling of the field to the waveguide mode of the aperture, which is naturally diameter dependent. Thereby we provide a complete description of the radiation by holes in the optical regime that involves both vectorial aspects of diffraction and the real properties of the metal screen.

Our experiments consist of measuring the angular distribution of the light diffracted through single holes. To this aim, we have designed a high-precision goniometry setup, described in detail in the Supplemental Material [23]. We used $300 \mathrm{~nm}(h)$ thick Ag films freely suspended or deposited on a glass substrate (thickness $1 \mathrm{~mm}$ ). Using a focused ion beam (FIB), circular holes were milled through the metal film. We have checked that both suspended and deposited films provided the same results, the latter ones being more convenient experimentally (silica substrate on the illumination side). The samples were positioned in the $x y$ plane of the goniometer [see Fig. 1(a)] and the hole carefully aligned along the optical axis of the setup. A single mode Gaussian beam emitted from a laser diode at a wavelength $\lambda=660 \mathrm{~nm}$ was linearly polarized and weakly focused at normal incidence on the metal surface by a microscope objective $(10 \times$, numerical aperture $\mathrm{NA}=0.3$ ), providing a compromise between plane-wave excitation and sufficient excitation density. The transmitted light was then collected in the far field with a multimode fiber coupled to a spectrometer. The latter behaved like a bandpass filter, where only photons detected in a small window around $\lambda$ are recorded. The fiber tip is scanned from $\theta=-60^{\circ}$ to $+30^{\circ}$ in a plane perpendicular to the metal film and passing through the center of the hole. We checked that no depolarization was induced by the setup, and that usual scalar-type diffraction patterns from large holes $(k r \gg 1)$ were recovered [Fig. 2(a)]. Because of the
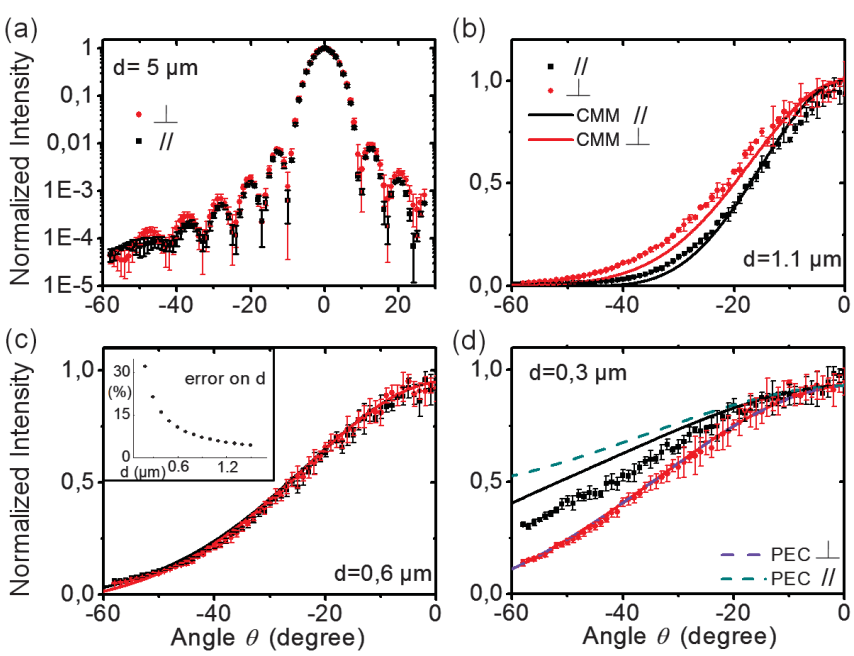

FIG. 2 (color). Diffraction pattern recorded from holes with diameters (a) $d=5 \mu \mathrm{m}(k r \gg 1)$, (b) $1.1 \mu \mathrm{m}$, (c) $0.6 \mu \mathrm{m}$, and (d) $0.3 \mu \mathrm{m}$ [a logarithmic scale is used in panel (a)]. Single apertures are illuminated with an incident electric field polarized along the $x$ axis and the diffraction angular pattern is measured scanning in the $y z$ plane ( $I_{\perp}$, red curves) and in the $x z$ plane ( $I_{\|}$, black curves). Solid lines correspond to theory (CMM) and points are experimental data [in (d), dashed lines correspond to PEC theory]. (Inset) Error on the diameter of a hole (\%), induced by nanofabrication (FIB milling).

weak transmitted signal through subwavelength holes, great care was taken to keep optical noise under control with good thermal and mechanical stability of the whole setup.

For the theoretical analysis, we have used the coupled mode method (CMM), described in [3,24], which is valid for opaque metal films, i.e., for film thickness larger than 2-3 times the skin depth $\delta=\lambda /\left[2 \pi \operatorname{Im}\left(\sqrt{\epsilon_{m}}\right)\right]$, where $\epsilon_{m}$ is the dielectric constant of the metal. The CMM relies on a modal expansion of the field, where the dielectric properties of the metal are approximately treated via the surface impedance boundary conditions. The radiation pattern for any hole diameter can be obtained within the CMM, provided enough waveguide modes in the hole are taken into account. However, for a sufficiently small hole diameter the transmission process is controlled by the fundamental waveguide mode $\left(\mathrm{TE}_{11}\right)$ alone. This has two important consequences. First, it justifies that the experiments are performed with an illumination only set at normal incidence, since the radiation patterns of such small holes do not depend on the angle of incidence in this regime. Second, the radiation pattern, being then independent of the details of the relative amplitudes of the different waveguide modes, is accurately represented by the CMM. Moreover, the scattering cross section $\sigma(\theta, \phi)$ (power flux per solid angle along the direction defined by $\theta$ and $\phi$ ) can be computed analytically. For a circular hole, using polar coordinates and defining the $x$ axis $\left(\phi=0^{\circ}\right)$ as pointing in the direction of the in-plane component of the incident field, we obtain (see Supplemental Material [23] 
for details)

$$
\sigma(\theta, \phi)=\sigma_{0}\left[I_{p}(\theta) \cos ^{2}(\phi)+I_{s}(\theta) \sin ^{2}(\phi)\right],
$$

where the normalized angular distributions from $p$ and $s$ polarizations of the radiative modes are given by

$$
\begin{gathered}
I_{p}(\theta)=\frac{\left|1+z_{s}\right|^{2} \cos ^{2}(\theta)}{\left|\cos (\theta)+z_{s}\right|^{2}} \frac{4 J_{1}^{2}(\Phi)}{\Phi^{2}}, \\
I_{s}(\theta)=\frac{\left|1+z_{s}\right|^{2} \cos ^{2}(\theta)}{\left|1+z_{s} \cos (\theta)\right|^{2}} \frac{4 J_{1}^{\prime 2}(\Phi)}{\left(1-\Phi^{2} / u^{2}\right)^{2}} .
\end{gathered}
$$

$\Phi=k r \sin \theta$ and $u \approx 1.84$ is the first root of $J_{1}(u)=0$. The metal properties are characterized by the surface impedance $z_{s}=1 / \sqrt{\epsilon_{m}}$. The normalization factor $\sigma_{0}$ is a factor that controls the total transmittance but does not affect the radiation pattern. It is equal to $\sigma_{0}=k^{2} r^{2}$ $\left[4 \pi\left(u^{2}-1\right)\right]^{-1}\left|E_{11}^{\prime}\right|^{2}\left|1+z_{s}\right|^{-2}$, where $E_{11}^{\prime}$ is the amplitude of the $\mathrm{TE}_{11}$ mode at the hole opening [24]. $E_{11}^{\prime}$ is a function of $\lambda, r$, the metal thickness and the dielectric constant of the substrate. Note that $I(\theta=0, \phi)=I_{p}(0)=$ $I_{s}(0)=1$. Experimentally, the contributions from different polarizations can be independently measured by scanning along the $x z$ plane, when $I\left(\theta, \phi=0^{\circ}\right) \equiv I_{\|}(\theta)=I_{p}(\theta)$, and the $y z$ plane, when $I\left(\theta, \phi=90^{\circ}\right) \equiv I_{\perp}(\theta)=I_{s}(\theta)$. With these analytical expressions, it is in principle possible to determine the total transmittance through a single hole by the measurement of the intensity flux in a single direction.

We have checked the validity of these expressions through comparison against numerical calculations performed with the Green's dyadic (GD) method [25]. This comparison was restricted to diameters smaller than about one wavelength as, for larger diameters, the large number of mesh points required in the GD calculations makes this method impractical. In the considered range, the analytical expressions (1)-(3) provided an excellent agreement with the numerical results, especially when the penetration of the electromagnetic field in the lateral walls defining the hole was phenomenologically taken into account by enlarging the hole radius by one skin depth. The effective radius of the hole used hereafter is thus $r+\delta$. Based on the properties of waveguide modes, we can expect the expressions (1)-(3) to be valid below the cutoff wavelength of the second waveguide mode $\mathrm{TM}_{01}$ for arbitrary illumination (which, considering the metal as a PEC, can be estimated to occur at $d_{01} \sim \lambda / 1.3$, i.e., $d_{01} \sim 504 \mathrm{~nm}$ for $\lambda=660 \mathrm{~nm}$ ). However, for normal incidence only $\mathrm{TE}_{1 n}$ modes are excited and the range of validity is extended to the spectral region in which the $\mathrm{TE}_{12}$ mode is evanescent, i.e., $d \lesssim 1.7 \lambda(d \lesssim 1120 \mathrm{~nm}$ for $\lambda=660 \mathrm{~nm})$.

The different factors contributing to Eqs. (1)-(3) have a clear physical meaning. The first term in $I_{p}$ and $I_{s}$ gives the normalized intensity of an infinitesimal hole for each polarization. It depends on the finite dielectric constant of the real metal, and specially affects the radiation pattern of waves along the $\phi=0^{\circ}$ axis (associated with $I_{p}$ ). In this case and for real metals, the angular spectrum is reduced close to grazing radiation, in order to accommodate the SP mode associated with the creation of the pole in $I_{p}$ [26]. A factor $\cos (\theta)$ in this term arises from the projection of the current carried by each mode along the $z$ axis into the polar direction $(\theta, \phi)$. Apart from this, $\left|1+z_{s}\right|^{2}$ stems from the angular spectrum of electromagnetic states at the hole position. The second term in $I_{p}$ and $I_{s}$ deals with the geometry of the hole. It corresponds to the modulus square of the two-dimensional Fourier transform of the field at the hole area (given by $\mathrm{TE}_{11}$ mode). This factor has the same origin as the one appearing in the Fraunhofer approximation. Notice that Eqs. (1)-(3) recover known expressions for the radiation pattern in two limiting cases: (i) a finite-size hole in a thick PEC $\left(z_{s}=0\right)$ [16], or (ii) an infinitesimal hole in a real metal film [27], which is obtained by taking the limit $k r \rightarrow 0$ in Eqs. (2) and (3), i.e., replacing the second factor in $I_{p}$ and $I_{s}$ by unity (see the Supplemental Material [23] for further details).

Figure 1(c) presents the experimental variation of $\sigma(\theta=0, \phi)$ with respect to the hole diameter $d$, together with the CMM prediction (red curve). The agreement is very good, as expected from previous comparisons for this quantity between experimental results and the CMM calculations [15]. Note that $\sigma(\theta=0, \phi)$ depends on the absolute transmitted intensity, and therefore on both metal thickness and the propagation constant inside the hole. In what follows, we will study the quantity $I(\theta, \phi)=$ $\sigma(\theta, \phi) / \sigma_{0}$ which, according to the analytical expressions and GD calculations, only depends on the geometrical section of the hole at the exit side (see the Supplemental Material [23]). We will concentrate on the quantities $I_{\|}(\theta)$ and $I_{\perp}(\theta)$ which, as mentioned before, is a way of isolating the contributions from $I_{p}(\theta)$ and $I_{s}(\theta)$, respectively.

We show in Fig. 2 the normalized experimental diffraction patterns recorded for four diameters [2(a) $d=5 \mu \mathrm{m}, 2$ (b) $d=1.1 \mu \mathrm{m}, 2(\mathrm{c}) d=0.6 \mu \mathrm{m}$, and 2(d) $d=0.3 \mu \mathrm{m}]$ and, for the three last, a comparison with the angular patterns $I(\theta)$ given by the analytical expression in Eq. (1). In the calculations we have taken $\epsilon_{A g}=-17.0+0.99 i$ at $\lambda=660 \mathrm{~nm}$ [28]. It is clear from Figs. 2 and 3 that four regimes can be identified, depending on the relative contribution of $I_{\|}$with respect to $I_{\perp}$. A first regime can be identified as a pseudoscalar regime, occurring for with $d>2 \lambda$, where $I_{\perp}=I_{\|}$ [Figs. 2(a) and 3(a), region 1]. This is not discussed theoretically in this paper because it is out of the domain of validity of our model (in the single-mode approximation), and it is well described by models based on Kirchhoff's scalar theory. Then in the region where $\lambda<d<2 \lambda$, there is a difference in intensity between measurements performed in the $y z$ plane and the $x z$ plane, with $I_{\perp}$ intensity mainly above the $I_{\|}$one [Figs. 2(b) and 3(a), region 2]. The third regime, illustrated by Figs. 2(c) and 3(a), region 3, presents a different 

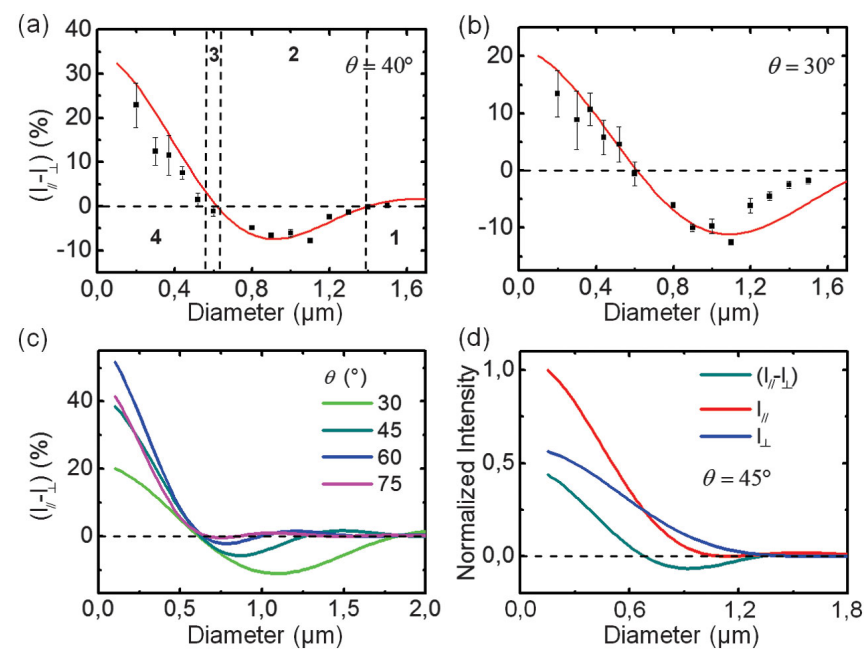

FIG. 3 (color). Normalized difference between $I_{\|}$and $I_{\perp}$ angular distributions at a fixed angle of analysis [(a) $\theta=40^{\circ}$ and (b) $\theta=30^{\circ} \mathrm{]}$, for different hole diameters. Black squares are experimental data and solid red lines correspond to theory. Region 1 corresponds to the scalar regime $d>2 \lambda$, region 2 is equivalent to $\lambda<d<2 \lambda$, region 3 to $d \sim \lambda$, and region 4 to $d<\lambda$. (c) The same theoretical normalized differences versus $d$, at different angles of detection $(\theta)$. (d) Theoretical evolution of $I_{\|}, I_{\perp}$, and their normalized difference versus hole diameter $d$, at $\theta=45^{\circ}$.

behavior with $I_{\perp}=I_{\|}$for $d \sim \lambda$. Then finally, a fourth regime [Figs. 2(d) and 3(a), region 4] arises for $d<\lambda$, where $I_{\perp}<I_{\|}$, which is completely coherent with previous experimental results $[9,21]$ despite the tip geometry and metal thickness in those experiments. The error bars in the experimental curves correspond to several measurements done with holes milled with identical parameters, i.e., identical effective diameters. Errors on the diameter, due to milling, are shown in the inset of Fig. 2(c). Obviously, this error is small at large diameter, and becomes important for subwavelength holes. Figures 2(b) and 2(c) show very good agreement between experiment and theory for both scanning orientations. For smaller holes, as in Fig. 2(d), the agreement is still good for $I_{\perp}$. For $I_{\|}$however, and even if the global shapes are really close, theory seems to slightly overestimate the intensity. The small difference between CMM and data for $I_{\perp}$ can be explained by the uncertainty in the diameter shown in the inset of Fig. 2(c), knowing that a scan in the $y z$ plane is less sensitive to this parameter. More important to stress is the difference at large angles in $I_{\|}$ between PEC and Ag. As discussed above, in a real metal the radiation pattern close to grazing angles in a parallel scan contains signatures of the existence of SP excitations [29], not present in a PEC.

Our theoretical formalism also reproduces the transition regime observed at $d \sim \lambda$. Figures 3(a) and 3(b) present a comparison between experimental and theoretical $\Delta I \equiv$ $\left(I_{\|}-I_{\perp}\right)$, for $\theta=40^{\circ}$ and $30^{\circ}$, both presenting the four regimes just discussed in good agreement with theory. This is seen by the calculations rendered in Fig. 3(c), where the (a)

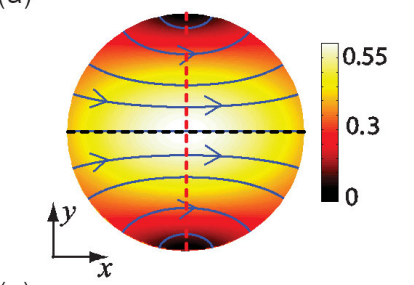

(c)

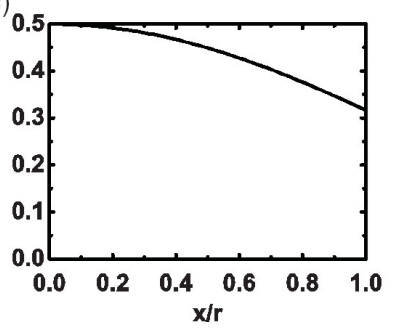

(d)

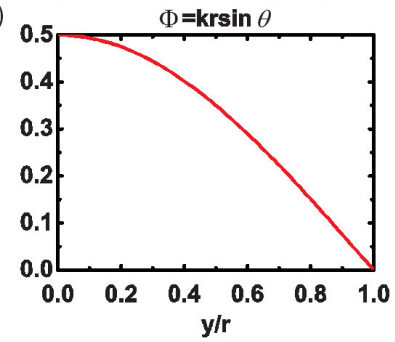

FIG. 4 (color). (a) Electric-field amplitude spatial distribution of the $\mathrm{TE}_{11}$ mode. (b) Fourier transform of $E_{x}(0, y)$ (red curve) and $E_{x}(x, 0)$ (black curve). (c) Crosscut of $E_{x}$ along the black dashed line in (a). (d) Crosscut of $E_{y}$ along the red dashed line in (a).

$d \sim \lambda$ transition is predicted even at moderate angles. As explained by a simple analysis of the model, this transition occurs because radiation is governed by two factors.

The first factor is the coupling to radiation modes in the scanning plane, which involves the Fourier transform of the field at the hole along the relevant direction. When the scanning plane is the $x z$ plane, this direction is the $x$ axis, while for the scanning plane in the $y z$ plane, it is the Fourier transform along the $y$ direction that matters. As illustrated in Fig. 4, the electric field of the relevant $\mathrm{TE}_{11}$ mode points preferentially along the $x$ direction and therefore, it is more confined along the $y$ direction than along the $x$ direction. The coupling is thus better for large in-plane wave vectors $\left(k_{\|}>1 / r\right)$ along the $y z$ plane than along the $x z$ plane, as it can be understood from a simple Fourier argument relating the near-field modal distribution to the far field. For small holes this is not important, as these large wave vectors are outside the light cone, but for larger holes this mechanism impairs radiation along the $x z$ plane [see Fig. 4(b)].

The second factor is related to the important point that, as shown by the first term in Eqs. (2) and (3), the angular spectrum at the metal surface with in-plane component of the wave vector is different depending of whether the scan is performed in the $x z$ or $y z$ plane. For a scan in the $y z$ plane, the angular spectrum goes as $\cos (\theta)$, while for a scan in the $x z$ plane, it increases as $1 / \cos (\theta)$ (except for the mentioned decay close to grazing radiation). As a consequence, radiation is impaired this time along the $y z$ plane.

We therefore have two competing factors but, while the strength of the first one depends on hole size, the second one does not. The fact that the different mechanisms dominate for different regions in parameter space is the reason for the existence of the transition which is experimentally found at $d \sim \lambda$. 
The crossover that occurs when $I_{\|}=I_{\perp}$ is largely independent of the angle of observation, as shown in Figs. 2(b) and 3(c), and it is also largely independent on the dielectric constant of the metal. Therefore, the critical diameter can be estimated for a particular value of $\theta$ for a PEC. For an analytical estimation it is convenient to take the limit $\Phi \rightarrow u$ in both $I_{\perp}$ and $I_{\|}$. We have $I_{\perp}=\left[\left(u^{2}-1\right)^{2}\right.$ $\left.\cos ^{2} \theta\right] J_{1}^{2}(u) / u^{2}$ and $I_{\|}=4 J_{1}^{2}(u) / u^{2}$. Thanks to the common term $J_{1}^{2}(u) / u^{2}$, we find that $I_{\|}=I_{\perp}$ when the condition $\left(u^{2}-1\right)^{2} \cos ^{2} \theta=4$ is fulfilled. It occurs for the angle $\theta=\cos ^{-1}\left[2\left(u^{2}-1\right)^{-1}\right] \approx 33^{\circ}$ and $d / \lambda=\pi^{-1} u\left(u^{2}-1\right)$ $\left[\left(u^{2}-1\right)^{2}-4\right]^{-1 / 2} \approx 1.07$, in agreement with the experimental observation that the transition occurs for $d \approx \lambda$.

In conclusion, we have presented a complete experimental and theoretical study of diffraction properties of circular apertures in a real, thick metallic screen, with dimension varying from scalar to subwavelength regimes. We obtained a good agreement between measured diffraction patterns and theoretical predictions of the modal expansion technique for the radiation pattern, cast into a simple analytical form which takes into account both the finite hole size and the dielectric properties of real metals. The observed transition occurring at $d \sim \lambda$ for all scanning angles characterized by the sign inversion of the difference $I_{\|}-I_{\perp}$ is consistently explained by two competing factors, one depending on the hole diameter, the other on the dependency on scanning orientations of the angular spectrum of electromagnetic modes. This study draws a relation between scalar and vectorial descriptions providing a deep understanding of single subwavelength holes, one of the most fundamental but subtle systems in optics.

We acknowledge support from the ERC (Grant 227557), the Spanish Ministry of Science and Innovation (projects MAT2008-06609-C02 and CSD2007-046-Nanolight.es), and the Chinese Scholarship Council (CSC).

[1] E. Betzig and J. K. Trautman, Science 257, 189 (1992).

[2] C. Genet and T. W. Ebbesen, Nature (London) 445, 39 (2007).

[3] F. J. García-Vidal, L. Martín-Moreno, T. W. Ebbesen, and L. Kuipers, Rev. Mod. Phys. 82, 729 (2010).

[4] H. J. Lezec, A. Degiron, E. Devaux, R. A. Linke, L. Martín-Moreno, F. J. García-Vidal, and T.W. Ebbesen, Science 297, 820 (2002).
[5] M. J. Levene, J. Korlach, S. W. Turner, M. Foquet, H. G. Craighead, and W. W. Webb, Science 299, 682 (2003).

[6] H. Rigneault, J. Capoulade, J. Dintinger, J. Wenger, N. Bonod, E. Popov, T. W. Ebbesen, and P.-F. Lenne, Phys. Rev. Lett. 95, 117401 (2005).

[7] M. L. Juan, R. Gordon, Y. Pang, F. Eftekhari, and R. Quidant, Nature Phys. 5, 915 (2009).

[8] H. W. Kihm et al., Nature Commun. 2, 451 (2011).

[9] C. Obermüller and K. Karrai, Appl. Phys. Lett. 67, 3408 (1995).

[10] R. Wannemacher, Opt. Commun. 195, 107 (2001).

[11] F. J. Garcia de Abajo, Opt. Express 10, 1475 (2002).

[12] A. Degiron, H. J. Lezec, N. Yamamoto, and T. W. Ebbesen, Opt. Commun. 239, 61 (2004).

[13] E. Popov, M. Nevière, A. Sentenac, N. Bonod, A.-L. Fehrembach, J. Wenger, P.-F. Lenne, and H. Rigneault, J. Opt. Soc. Am. A 24, 339 (2007).

[14] J. Kindler, P. Banzer, S. Quabis, U. Peschel, and G. Leuchs, Appl. Phys. B 89, 517 (2007).

[15] F. Przybilla, A. Degiron, C. Genet, T. W. Ebbesen, F. de León-Pérez, J. Bravo-Abad, F. J. García-Vidal, and L. Martín-Moreno, Opt. Express 16, 9571 (2008).

[16] J. D. Jackson, Classical Electrodynamics (John Wiley \& Sons, New York, 1999), 3rd ed.

[17] H. A. Bethe, Phys. Rev. 66, 163 (1944).

[18] C. J. Bouwkamp, Philips Res. Rep. 5, 321 (1950).

[19] Y. Leviatan, J. Appl. Phys. 60, 1577 (1986).

[20] A. Roberts, J. Opt. Soc. Am. A 4, 1970 (1987).

[21] D. J. Shin, A. Chavez-Pirson, S. H. Kim, S. T. Jung, and Y. H. Lee, J. Opt. Soc. Am. A 18, 1477 (2001).

[22] A. Drezet, J. C. Woehl, and S. Huant, Europhys. Lett. 54, 736 (2001).

[23] See Supplemental Material http://link.aps.org/ supplemental/10.1103/PhysRevLett.109.023901 for a detailed discussion of the experimental setup and the theoretical method.

[24] F. de León-Pérez, G. Brucoli, F. J. García-Vidal, and L. Martín-Moreno, New J. Phys. 10, 105017 (2008).

[25] J. Alegret, P. Johansson, and M. Käll, New J. Phys. 10, 105004 (2008).

[26] A. Y. Nikitin, S. G. Rodrigo, F. J. García-Vidal, and L. Martín-Moreno, New J. Phys. 11, 123020 (2009).

[27] A. Y. Nikitin, F. J. García-Vidal, and L. Martín-Moreno, Phys. Rev. Lett. 105, 073902 (2010).

[28] P. B. Johnson and R. W. Christy, Phys. Rev. B 6, 4370 (1972).

[29] E. Popov, N. Bonod, M. Nevière, H. Rigneault, P.-F. Lenne, and P. Chaumet, Appl. Opt. 44, 2332 (2005). 